\title{
Himenópteros parasitóides (Insecta, Hymenoptera) coletados em cultura de soja (Glycine max (L.)) Merril (Fabaceae), no município de Nuporanga, SP, Brasil
}

\author{
Nelson Wanderley Perioto ${ }^{1}$ \\ Rogéria Inês Rosa Lara ${ }^{1}$ \\ José Carlos Carvalho dos Santos ${ }^{1}$ \\ Tatiane Cardoso da Silva ${ }^{2}$
}

\begin{abstract}
Parasitoid Hymenoptera collected in a soybean plantation (Glycine max (L.)) Merril (Fabaceae) at the municipal district of Nuporanga, SP, Brazil. Parasitoid Hymenoptera were collected by using Moericke trap placed in a soybean plantation (Glycine $\max$ (L.) Merril) (Fabaceae) of the variety Conquista, during the period of March $24^{\text {th }}$ to April $7^{\text {th }}$, 2000. A total of 4,969 specimens of parasitoids, belonging to seven superfamilies and 15 families, were collected. Scelionidae, Encyrtidae, Aphelinidae and Trichogrammatidae were the most common families, being responsible for $41.66 \%, 19.42 \%, 11.19 \%$ and $7.35 \%$ of the total number of parasitoids collected, respectively. Other eleven families showed the relative frequency lower than $5 \%$.
\end{abstract}

KEYwords. Brazil; Hymenoptera; parasitoid; soybean; survey.

\section{INTRODUÇÃO}

O presente estudo tem como objetivo reconhecer as famílias de himenopteros parasitóides e suas freqüências relativas em uma cultura comercial de soja, na safra de 1999/2000. Trabalho semelhante foi realizado porPeRIOTO et al. (2002), em cultura de algodão, no município de Ribeirão Preto, SP.

\section{MATERIAL E MÉTODOS}

Este ensaio foi realizado em uma área de $8.400 \mathrm{~m} 2$ de uma cultura comercial de soja, Glycine max (L.) Merril, (Fabaceae) variedade Conquista, instalada em Nuporanga, SP, no período de 24 de março a sete de abril de 2000. A cultura foi semeada em meados do mês de dezembro de 1999 com espaçamento de 0,5 m entre linhas e, quando do início do experimento, encontravase no estágio R 5.5. A metodologia utilizada assemelha-se à descrita em PeRIoto et al. (2002), dela diferindo apenas na altura em que foram dispostas as armadilhas, localizadas a $0,3 \mathrm{~m}$ e 1,0 $\mathrm{m}$ acima do nível do solo. Foi realizada uma aplicação de inseticidas no dia 24 de março de 2000, devido à ocorrência de pragas que ameaçavam a continuidade do experimento.

O material coletado foi depositado na Coleção Entomológica “Adolph Hempel” do Instituto Biológico, em São Paulo.

\section{RESULTADOS EDISCUSSÃO}

No presente estudo foram coletados 4.969 exemplares de himenópteros parasitóides pertencentes a 15 famílias, distribuídas em sete superfamílias (Tabela 1). Comparando-se estes resultados com aqueles obtidos por Perioto (1991), Perioto et al. (2002), Azevedo \& Santos (2000), Azevedo et al. (2002) e DALL'OGLIO et al. (2000) observa-se que, de forma geral, tem sido amostrado um maior número de superfamílias de himenópteros parasitóides em áreas de vegetação nativa que em agroecossistemas monoculturais. De acordo com dados obtidos por aqueles autores, nas coletas realizadas em áreas de Mata Atlântica, em vegetação de cerrado e em um transecto de cultura de eucalipto/mata nativa, o número de famílias de himenópteros parasitóides representadas variou de 25 a 30 , enquanto que em agroecossistemas monoculturais, como nas culturas de algodão e no presente estudo, este número foi de

1. Instituto Biológico, Laboratório de Sanidade Animal e Vegetal de Ribeirão Preto. Rua Peru, 1472-A, 14075-310 Ribeirão Preto-SP, Brasil. Endereço eletrônico: nperioto@biologico.br

2. Estagiária. Graduanda em Engenharia Agronômica do Centro Universitário "Moura Lacerda". Ribeirão Preto-SP, Brasil 
Tabela 1. Número de himenópteros parasitóides coletados nas armadilhas superiores (AS) e inferiores (AI), seu total e suas freqüências relativas em cultura de soja, na safra 1999/2000, em Nuporanga (SP).

\begin{tabular}{lrrrrrrrrrrr}
\hline & \multicolumn{1}{c}{ S } & \multicolumn{1}{c}{ T } & \multicolumn{1}{c}{ FRS } & FRI & \multicolumn{1}{c}{ FRTS } & FRTI & FRTS+I & FRSS & FRSI & FRSS+I \\
\hline Ceraphronoidea & $\mathbf{7 9}$ & $\mathbf{7 7}$ & $\mathbf{1 5 6}$ & $\mathbf{5 0 , 6 4}$ & $\mathbf{4 9 , 3 6}$ & $\mathbf{2 , 6 4}$ & $\mathbf{3 , 9 0}$ & $\mathbf{3 , 1 4}$ & & & \\
Chalcidoidea & $\mathbf{1 3 9 7}$ & $\mathbf{8 0 5}$ & $\mathbf{2 2 0 2}$ & $\mathbf{6 3 , 4 4}$ & $\mathbf{3 6 , 5 6}$ & $\mathbf{4 6 , 6 4}$ & $\mathbf{4 0 , 7 8}$ & $\mathbf{4 4 , 3 1}$ & & & \\
Aphelinidae & 282 & 274 & 556 & 50,72 & 49,28 & 9,42 & 13,88 & 11,19 & 20,19 & 34,04 & 25,25 \\
Eulophidae & 58 & 19 & 77 & 75,32 & 24,68 & 1,94 & 0,96 & 1,55 & 4,15 & 2,36 & 3,50 \\
Encyrtidae & 725 & 240 & 965 & 75,13 & 24,87 & 24,21 & 12,16 & 19,42 & 51,90 & 29,81 & 43,82 \\
Chalcididae & 16 & 31 & 47 & 34,04 & 65,96 & 0,53 & 1,57 & 0,95 & 1,15 & 3,85 & 2,13 \\
Mymaridae & 108 & 56 & 164 & 65,85 & 34,15 & 3,61 & 2,84 & 3,30 & 7,73 & 6,96 & 7,45 \\
Pteromalidae & 6 & 21 & 27 & 22,22 & 77,78 & 0,20 & 1,06 & 0,54 & 0,43 & 2,61 & 1,23 \\
Torymidae & 1 & 0 & 1 & 100,00 & 0,00 & 0,03 & 0,00 & 0,02 & 0,07 & 0,00 & 0,05 \\
Trichogrammatidae & 201 & 164 & 365 & 55,07 & 44,93 & 6,71 & 8,31 & 7,35 & 14,39 & 20,37 & 16,58 \\
Chrysidoidea & $\mathbf{3 1}$ & $\mathbf{2 8}$ & $\mathbf{5 9}$ & $\mathbf{5 2 , 5 4}$ & $\mathbf{4 7 , 4 6}$ & $\mathbf{1 , 0 4}$ & $\mathbf{1 , 4 2}$ & $\mathbf{1 , 1 9}$ & & & \\
Bethylidae & 31 & 28 & 59 & 52,54 & 47,46 & 1,04 & 1,42 & 1,19 & & & \\
Cynipoidea & $\mathbf{6 6}$ & $\mathbf{6 4}$ & $\mathbf{1 3 0}$ & $\mathbf{5 0 , 7 7}$ & $\mathbf{4 9 , 2 3}$ & $\mathbf{2 , 2 0}$ & $\mathbf{3 , 2 4}$ & $\mathbf{2 , 6 2}$ & & & \\
Eucoilidae & 66 & 64 & 130 & 50,77 & 49,23 & 2,20 & 3,24 & 2,62 & & & \\
Ichneumonoidea & $\mathbf{8 4}$ & $\mathbf{8 4}$ & $\mathbf{1 6 8}$ & $\mathbf{5 0 , 0 0}$ & $\mathbf{5 0 , 0 0}$ & $\mathbf{2 , 8 0}$ & $\mathbf{4 , 2 6}$ & $\mathbf{3 , 3 8}$ & & & \\
Braconidae & 39 & 49 & 88 & 44,32 & 55,68 & 1,30 & 2,48 & 1,77 & 46,43 & 58,33 & 52,38 \\
Ichneumonidae & 45 & 35 & 80 & 56,25 & 43,75 & 1,50 & 1,77 & 1,61 & 53,57 & 41,67 & 47,62 \\
Platygastroidea & $\mathbf{1 2 7 9}$ & $\mathbf{8 8 3}$ & $\mathbf{2 1 6 2}$ & $\mathbf{5 9 , 1 6}$ & $\mathbf{4 0 , 8 4}$ & $\mathbf{4 2 , 7 0}$ & $\mathbf{4 4 , 7 3}$ & $\mathbf{4 3 , 5 1}$ & & & \\
Scelionidae & 1223 & 847 & 2070 & 59,08 & 40,92 & 40,83 & 42,91 & 41,66 & 95,62 & 96,83 & 96,11 \\
Platygasteridae & 56 & 28 & 84 & 66,67 & 33,33 & 1,87 & 1,42 & 1,69 & 4,38 & 3,17 & 3,89 \\
Proctotrupoidea & $\mathbf{5 9}$ & $\mathbf{3 3}$ & $\mathbf{9 2}$ & $\mathbf{6 4 , 1 3}$ & $\mathbf{3 5 , 8 7}$ & $\mathbf{1 , 9 7}$ & $\mathbf{1 , 6 7}$ & $\mathbf{1 , 8 5}$ & & & \\
Diapriidae & 59 & 33 & 92 & 64,13 & 35,87 & 1,97 & 1,67 & 1,85 & & & \\
\hline TOTAL & $\mathbf{2 9 9 5}$ & $\mathbf{1 9 7 4}$ & $\mathbf{4 9 6 9}$ & & & & & & & \\
\hline & & & & & & & & & & \\
\hline
\end{tabular}

S e I= número de himenópteros parasitóides coletados nas armadilhas superiores (AS) e inferiores (AI), respectivamente; T= $\Sigma$ S+I; FRS e FRI= freqüência relativa dos himenópteros parasitóides coletados nas AS e AI, respectivamente; FRPS e FRPI= freqüência relativa das superfamílias e famílias de himenópteros parasitóides coletados nas AS e AI, respectivamente, em relação ao total de himenópteros parasitóides coletados; FRPS+I= $\Sigma$ FRPS+FRPI; FRSS e FRSI = freqüência relativa das famílias de himenópteros parasitóides coletados nas AS e AI, respectivamente, em relação ao total de himenópteros parasitóides da superfamília a que pertencem; FRSS+I= $\Sigma$ FRSS+FRSI.

19 e 15 , respectivamente.

As famílias mais freqüentemente amostradas neste estudo foram Scelionidae (Platygastroidea), Encyrtidae, Aphelinidae e Trichogrammatidae (Chalcidoidea) que representaram 41,66\%, $19,42 \%, 11,19 \%$ e $7,35 \%$, respectivamente, do número total de himenópteros parasitóides coletados. As quatorze famílias restantes apresentaram freqüências relativas inferiores a 5\%.

Dentre os Platygastroidea, destacou-se a família Scelionidae, com $95,07 \%$ do total de exemplares coletados nesta superfamília. Fez-se notar a presença dos gêneros Trissolcus Ashmead, 1893 e Telenomus Haliday, 1833 que, conjuntamente, foram responsáveis por $87,97 \%$ dos Scelionidae e $36,65 \%$ do total de himenópteros parasitóides capturados. Deve-se ressaltar que espécies pertencentes a esses gêneros são importantes agentes de controle natural do complexo de percevejos-praga da soja.

Foram coletados himenópteros parasitóides de oito famílias pertencentes à superfamília Chalcidoidea, dentre as quais destacaram-se as famílias Encyrtidae, Aphelinidae, Trichogrammatidae e Mymaridae que representaram, respectivamente, $43,82 \%, 25,25 \%, 16,58 \%$ e $7,45 \%$ do total de himenópteros parasitóides pertencentes àquela superfamília. Na família Encyrtidae, destacou-se uma espécie não identificada do gênero Copidosoma Ratzeburg, 1844, que representou $90,36 \%$ do total de encirtídeos e $17,55 \%$ do total de himenópteros parasitóides coletados. Provavelmente, a espécie por nós obtida é Copidosoma floridanun (Ashmead, 1900), relatada por Moraes et al. (1991) como parasitóide de Pseudoplusia includens (Walker, 1857) (Lepidoptera: Noctuidae). P. includens é uma importante praga do complexo de desfolhadores da cultura da soja. As famílias Trichogrammatidae e Mymaridae representaram, conjuntamente, $24,03 \%$ do total de Chalcidoidea parasitóides coletados. A alta frequiência relativa destas famílias pode ser atribuída à existência de grande número de hospedeiros potenciais destas famílias na cultura em questão.

No que se refere à distribuição altitudinal dos himenópteros parasitóides capturados, observou-se que $60,27 \%$ do total daqueles insetos foram coletados nas armadilhas de Moericke colocadas a $1 \mathrm{~m}$ em relação ao nível do solo. Este padrão, à exceção das famílias Chalcididae e Pteromalidae (Chalcidoidea) e Braconidae (Ichneumonoidea) que tiveram mais de 50\% dos exemplares coletados nas armadilhas de Moericke colocadas a 0,3 m em relação ao nível do solo, foi observado para as demais famílias capturadas. 


\section{REFERENNCIAS}

Azevedo, C. O. \& H. S. Santos. 2000. Perfil da fauna de himenópteros parasitóides (Insecta, Hymenoptera) em uma área de Mata Atlântica da Reserva Biológica de Duas Bocas, Cariacica, ES, Brasil. Boletim do Museu de Biologia Mello Leitão, série nova, 11/12: 116126.

Azevedo, C. O.; R. Kawada; M. T. Tavares \& N. W. Perioto. 2002. Perfil da fauna de himenópteros parasitóides (Insecta, Hymenoptera) em uma área de Mata Atlântica do Parque Estadual da Fonte Grande, Vitória,
ES, Brasil. Revista Brasileira de Entomologia 46(2):133-137.

Dall'Oglio, O. T.; J. C. Zanuncio; C. O. Azevedo \& A. G. B. Medeiros. 2000. Survey of the Hymenoptera Parasitoids in Eucalyptus grandis and in a native vegetation area in Ipara, State of Minas Gerais, Brazil. Anais da Sociedade Entomológica do Brasil 20(3): 583588.

Perioto, N. W.; R. I. R. Lara; J. C. C. Santos \& A. Selegatto. 2002. Himenópteros parasitóides coletados em cultura de algodão (Gossypiun hirsutun L.) (Malvaceae), no município de Ribeirão Preto, SP, Brasil. Revista Brasileira de Entomologia 46(2):165-168. 